\title{
A Rapid Screening Test on Dried Blood for the Neonatal Diagnosis of Tyrosinemia Type I
}

\author{
Farahnaz Bodaghkhan, ${ }^{1}$ Bita Geramizadeh, ${ }^{1,2,}{ }^{*}$ Abbas Abdollah Rajeh, ${ }^{2}$ Mahmoud Haghighat, ${ }^{3,4}$ \\ Mohsen Dehghani, ${ }^{1,3,4}$ Naser Honar, ${ }^{3,4}$ Mojgan Zahmatkeshan, ${ }^{3,4}$ and Mohammad-Hadi Imanieh ${ }^{3,4}$ \\ ${ }^{1}$ Department of Pathology, Shiraz University of Medical Sciences, Shiraz, IR Iran \\ ${ }^{2}$ Transplant Research Center, Shiraz University of Medical Sciences, Shiraz, IR Iran \\ ${ }^{3}$ Department of Pediatrics, Shiraz University of Medical Sciences, Shiraz, IR Iran \\ ${ }^{4}$ Gastroenterology Research Center, Shiraz University of Medical Sciences, Shiraz, IR Iran \\ "Corresponding author: Bita Geramizadeh, Department of Pathology, Shiraz University of Medical Sciences, Shiraz, IR Iran. E-mail: geramib@gmail.com
}

Received 2015 November 07; Revised 2015 December 13; Accepted 2015 December 22.

\begin{abstract}
Background: Tyrosinemia is an inherited metabolic disorder characterized by elevated levels of tyrosine and its metabolites in plasma. Without treatment, the disease will progress to hepatic and renal failure, so that without liver transplantation will cause death in less than 10 years of age. So, early diagnosis and treatment can be life saving and crucial. It means that with early treatment starting in the neonatal period, the patient can have normal life with very few restrictions in diets containing tyrosine and phenylalanine.

Objectives: In this study we wanted to evaluate an easy to perform, rapid and sensitive qualitative test with low cost, as a part of neonatal screening tests to help early diagnosis and treatment of hereditary tyrosinemia.

Patients and Methods: In this cross sectional study, during the study period (2013 - 2014), 100 patients were selected. Fifty three (53) of these patients had proven tyrosinemia and the other 47 cases biliary atresia, paucity of intrahepatic bile ducts, cytomegalovirus (CMV) hepatitis, galactosemia and storage diseases.

Results: There were 2 false negative and 14 false positive cases of hereditary tyrosinemia (HT-1) in the test. Six cases of biliary atresia, 7 cases of paucity of intrahepatic bile ducts and one patient with cytomegalovirus (CMV) hepatitis were falsely positive with the test. Sensitivity of the test was $96.23 \%$, specificity $71.43 \%$, positive predictive value (PPV) $78.46 \%$, and negative predictive value (NPV) $94.59 \%$. Conclusions: This rapid qualitative test on dried blood sample is an easy, cheap, and feasible method for the screening of hereditary tyrosinemia in neonatal period.
\end{abstract}

Keywords: Hereditary Tyrosinemia Type I, Succinylacetone, Screening Test, Newborn

\section{Background}

Tyrosinemia is an autosomal recessive hereditary disease resulting from elevated tyrosine in the blood. This disease is genetically and clinically divided into three types: type I, type II, and type III. These three types are different with respect to clinical manifestations and metabolic underlying enzymatic causes (1).

Tyrosinemia type I or hepatorenal tyrosinemia, the most severe form of this disorder, is caused by a genetic deficiency of fumarylacetoacetate hydrolase (FAH). Tyrosinemia type II or oculocutaneous tyrosinemia is caused by a deficiency of the enzyme tyrosine aminotransferase. Tyrosinemia type III or 4-hydroxyphenylpyruvate dioxygenase deficiency occurs because of an enzyme deficiency of 4-hydroxylphenylpyruvate dioxygenase (2).

Tyrosinemia type I causes development of severe liver disease in infancy, hypophosphatemic rickets, and neurologic crises. If left untreated, most patients die of liver failure in the first years of life, however treatment with 2(2-nitro-4-trifluoromethylbenzoyl)-1, 3-cyclohexanedione (NTBC; Nitisinone) has become available since 10 years ago. It is much more effective when initiated early in life, which emphasizes on the importance of development of screening tests, to identify affected patients by newborn screening (3).

\section{Objectives}

In this study, we tried to evaluate a rapid screening test on dried blood samples in neonates with different kinds of neonatal diseases including proven tyrosinemia type I, and compared the results of screening test with final diagnosis. 


\section{Patients and Methods}

In this study, we collected whole blood samples from 100 neonates who have referred to our pediatric clinic during the study period (September 2013 - September 2014) from 0.5 to $180(15.9 \pm 29)$ days old. All the patients who have received any kind of treatment have been excluded from the study.

There were 48 girls and 52 boys. Between these case, 53 patients turned out to be tyrosinemia by confirmatory and golden standard methods (sum of urine succynil acetone, blood tyrosine level, alpha-feto protein, clinical manifestation, liver biopsies and other paraclinical findings put together), and 47 patients had proven other diseases such as biliary atresia, CMV hepatitis, paucity of intrahepatic bile ducts, etc. The blood collected by a heel-stick was spotted on filter paper. Succinylacetone was extracted from residual blood spots with a solution of acetonitrile, formic acid, hydrazine hydrate and 5, 7-dioxooctanoic acid. The micro-titration plates were agitated gently and incubated at $37^{\circ} \mathrm{C}$ covered with aluminum foil. After 45 minutes the extract was transferred to a second plate, which was covered with aluminum foil, for spectrophotometric microassay (4). The result of the test was compared with the final diagnosis by chi-square test.

\section{Results}

We tested 100 neonates, 53 of which were proven to have tyrosinemia and 47 patients had other diseases. Table 1 shows the demographic findings and frequency of these 100 neonates with various diseases and the results of the rapid screening test.

These patients had been presented with jaundice ( 40 patients), hepatosplenomegay (14 patients), and GI bleeding, coagulopathy, ascites, rickets, hypoglycemia and convulsion, generalized edema, etc.

Among the patients, there were 14 false positive tests and 2 false negative tests with the screening rapid method on dried blood. Among false positive cases, 6 cases were biliary atresia, 7 cases paucity of intrahepatic bile ducts, and 1 patient was proven to have neonatal CMV hepatitis.

According to above findings, this screening test has more false positive cases than false negatives (14 vs. 2 ), the sensitivity was $96.23 \%$, specificity $71.43 \%$, positive predictive value (PPV) $78.46 \%$, and negative predictive value $94.59 \%$.

\section{Discussion}

Tyrosinemia type I (HT-1) is reported to affect 1 in every 100,000 to 120,000 babies worldwide; however in our center which is the largest referral center of liver diseases in
South of Iran, the incidence seems to be much higher (5). It is an autosomal recessive disorder caused by mutations in the FAH gene that leads to deficiency of the fumarylacetoacetic hydrolase which is the last enzyme in the tyrosine degradation pathway (6).

The presence of succinyl acetone in urine or blood is the characteristic test for the diagnosis of tyrosinemia type I which if untreated and without liver transplantation, will be usually fatal before the age 10 years (7). Affected children may present with liver or kidney failure, neurological crisis, rickets, failure to thrive, and hepatocellular carcinoma (8). With early treatment and a few restrictions, most of the patients can survive with normal lives. The most popular treatment is composed of 2-(2-nitro-4-3 trifluoromethylbenzoyl)-1, 3-cyclohexanedione (NTBC) and a diet low in tyrosine and phenylalanine. Therefore early diagnosis and therapy can be very crucial in improving outcome (9).

Newborn screening for HT1 is not widely available in most areas of the world, may be because it has been considered as an uncommon disease. However as it has been mentioned, diagnosis and early treatment with NTBC and diet can be life saving. There have been very few studies that have proven newborn screening by testing succinylacetone (SA) on blood spot (10). Children with HT1 are mostly asymptomatic in the first days of life, so newborn screening is the only way for early diagnosis and initiation of treatment $(11,12)$. Determination of blood tyrosine level is neither specific nor sensitive (11). So, in this study, we evaluated 100 newborns with different clinical presentations under the age of 3 months based on determination of succynil acetone in the dried spot blood from the heel. The cost of the test is low and it can be performed easily without specific instruments or skills. This test has also been studied by Schulze et al. in 2001 (4) with acceptable results. It is a qualitative test with less than 4 hours procedure. In our study the sensitivity of the test was higher than 95\%, which is completely acceptable for being a screening test (13).

We suggest this qualitative test to be added in the screening program of neonates in Iran, because it can be easily performed without any additional sample in newborn screening program.

\section{Footnotes}

Authors' Contribution: Farahnaz Bodaghkhan: Setting up the test; Bita Geramizadeh: Setting up the test, designing the project, collecting the patients and writing the paper; Abbas Abdollah Rajeh: Collecting the data; Mahmoud Haghighat: Collecting the patients; Mohsen Dehghani: Collecting the patients; Naser Honar: Collecting 
Table 1. Frequency of Diseases in the 100 Patients who Have Been Tested by Rapid Method

\begin{tabular}{|c|c|c|c|c|c|c|}
\hline \multirow{2}{*}{ Disease } & \multirow{2}{*}{ Number } & \multicolumn{2}{|c|}{ Gender } & \multirow{2}{*}{ Age, $d$} & \multirow{2}{*}{ SA Test Positive ${ }^{a}$} & \multirow{2}{*}{ SA Test Negative ${ }^{a}$} \\
\hline & & Female & Male & & & \\
\hline Tyrosinemia & 53 & 23 & 30 & $3-45$ & $51(96.2)$ & $2(3.8)$ \\
\hline Biliary atresia & 20 & 10 & 10 & $0.5-54$ & $6(30)$ & $14(70)$ \\
\hline $\begin{array}{l}\text { Paucity of intrahepatic } \\
\text { bile ducts }\end{array}$ & 19 & 9 & 10 & $1-50$ & $7(36.8)$ & $12(63.2)$ \\
\hline $\begin{array}{l}\text { Glycogen storage } \\
\text { disease }\end{array}$ & 2 & 1 & 1 & $120-180$ & 0 & $2(100)$ \\
\hline Lipid storage disease & 2 & 1 & 1 & $90-150$ & 0 & $2(100)$ \\
\hline CMV hepatitis & 2 & 2 & 0 & $3-35$ & $1(50)$ & $1(50)$ \\
\hline Galactosemia & 2 & 2 & 0 & $0.5-32$ & 0 & $2(100)$ \\
\hline Total & 100 & \multicolumn{2}{|c|}{100} & 100 & $65(65)$ & $35(35)$ \\
\hline
\end{tabular}

${ }^{\mathrm{a}}$ Values are expressed as No. (\%).

the patients; Mojgan Zahmatkeshan: Collecting the patients; Mohammad-Hadi Imanieh: Collecting the patients.

Funding/Support: This manuscript has been supported by the project number 3378 of Shiraz University of Medical Sciences.

\section{References}

1. Nakamura K, Matsumoto S, Mitsubuchi H, Endo F. Diagnosis and treatment of hereditary tyrosinemia in Japan. Pediatr Int. 2015;57(1):37-40. doi: 10.1111/ped.12550. [PubMed: 25443793].

2. Dehghani SM, Haghighat M, Imanieh MH, Karamnejad H, Malekpour A. Clinical and para clinical findings in the children with tyrosinemia referring for liver transplantation. Int J Prev Med. 2013;4(12):1380-5. [PubMed: 24498493].

3. Turgeon C, Magera MJ, Allard P, Tortorelli S, Gavrilov D, Oglesbee D, et al. Combined newborn screening for succinylacetone, amino acids, and acylcarnitines in dried blood spots. Clin Chem. 2008;54(4):65764. doi: 10.1373/clinchem.2007.101949. [PubMed: 18281422].

4. Schulze A, Frommhold D, Hoffmann GF, Mayatepek E. Spectrophotometric microassay for delta-aminolevulinate dehydratase in driedblood spots as confirmation for hereditary tyrosinemia type I. Clin Chem. 2001;47(8):1424-9. [PubMed:11468232].

5. Golbahar J, Karamizadeh Z, Honardar Z. Selective screening of amino acid disorders in the south-west of Iran, Shiraz. J Inherit Metab Dis. 2002;25(6):519-21. [PubMed: 12555946].

6. Pass KA, Morrissey M. Enhancing newborn screening for tyrosinemia type I. Clin Chem. 2008;54(4):627-9. doi: 10.1373/clinchem.2008.103200. [PubMed: 18375485].
7. la Marca G, Malvagia S, Pasquini E, Cavicchi C, Morrone A, Ciani F, et al. Newborn Screening for Tyrosinemia Type I: Further Evidence that Succinylacetone Determination on Blood Spot Is Essential. JIMD Rep. 2011;1:107-9. doi:10.1007/8904_2011_24. [PubMed:23430836].

8. Sander J, Janzen N, Terhardt M, Sander S, Gokcay G, Demirkol M, et al. Monitoring tyrosinaemia type I: Blood spot test for nitisinone(NTBC). Clin Chim Acta. 2011;412(1-2):134-8. doi: 10.1016/j.cca.2010.09.027. [PubMed: 20883679].

9. Fernandez-Lainez C, Ibarra-Gonzalez I, Belmont-Martinez L, MonroySantoyo S, Guillen-Lopez S, Vela-Amieva M. Tyrosinemia type I: clinical and biochemical analysis of patients in Mexico. Ann Hepatol. 2014;13(2):265-72. [PubMed: 24552869].

10. de Laet C, Dionisi-Vici C, Leonard JV, McKiernan P, Mitchell G, Monti $\mathrm{L}$, et al. Recommendations for the management of tyrosinaemia type 1. Orphanet J Rare Dis. 2013;8:8. doi: 10.1186/1750-1172-8-8. [PubMed: 23311542].

11. Lam R, Armenta A, Kilic M, Karpen SJ, Scaglia F, Seu P, et al. Tyrosinemia. Liver Transpl. 2002;8(5):500-1. doi: 10.1053/jlts.2002.31744. [PubMed: 12004353].

12. De Jesus VR, Adam BW, Mandel D, Cuthbert CD, Matern D. Succinylacetone as primary marker to detect tyrosinemia type I in newborns and its measurement by newborn screening programs. Mol Genet Metab. 2014;113(1-2):67-75. doi: 10.1016/j.ymgme.2014.07.010. [PubMed: 25066104].

13. Adam BW, Hall EM, Meredith NK, Lim TH, Haynes CA, De Jesus VR, et al. Performance of succinylacetone assays and their associated proficiency testing outcomes. Clin Biochem. 2012;45(18):1658-63. doi: 10.1016/j.clinbiochem.2012.08.007. [PubMed: 22906829]. 